\title{
Intelligent Reverse Sensor for Electric Wheelchair
}

\author{
Sooraj Surendran ${ }^{1 *}$, Gnanaguru G. ${ }^{2}$ \\ ${ }^{1 *}$ Research and Development, Ostrich Mobility, Bangalore, India \\ ${ }^{2}$ Research and Development, Ostrich Mobility, Bangalore, India \\ *Corresponding Author: soorajsudhasurendran@gmail.com
}

Available online at: www.isroset.org

Received: 12/Nov/2017, Revised: 27/Nov/2017, Accepted: 14/Dec/2017, Published: 31/Dec/2017

\begin{abstract}
This research article focuses on the design and implementation of a microcontroller based Automatic Reverse Sensor for Electric Wheelchair. The purposed model presents a reliable and cost effective obstacle detection technique and thereby identifying the obstacles only in reverse motion. The system functions in accordance to the controlled inputs from the sensor to the microcontroller.
\end{abstract}

Keywords - Electric wheelchairs, E-vehicles, Ultrasonic range finder, Obstacle detection

\section{INTRODUCTION}

In India out of 121 crore population, 2.68 crore population are disabled, which is $2.21 \%$ of total population. Among the disabled population, $56 \%$ are males and $44 \%$ are females, with $20 \%$ of them having disability in movement [1], which strongly suggests for an alternative mode of transportation. Even though the introduction of electric wheelchairs met their needs, certain limitations like Obstacle detection in reverse movements is not yet addressed. This project models the obstacle detection in reverse motion and thus ensures a safe reverse motion.

The rest of the paper is organized as follows: Section 2 highlights the important closely associated works whereas the proposed architecture is explained in Section 3. The experimental set-up and the information flow are explained in Section 4. The important findings are consolidated in Section 5. The conclusions as well as future directions of this research work are elucidated in the section 6 . Finally, the section 7 provides the acknowledgement.

\section{RELATED WORK}

Reverse Sensor is quite a popular topic in electronic vehicular domain and it has been deployed with the following components, like reverse gear switches [2], complex algorithms with camera or image processing techniques [3], that detects the backward movement and gives notification to user when its proximity increases with the obstacle. The urge to initiate this project is to make a cost effective and reliable technique compared to the previous complex approaches, which paves their way for easy installation in any power wheelchairs for user safety

\section{METHODOLOGY}

The ultrasonic range finder is used to find the obstacle's relative distance with respect to the wheelchair in rear and that information is processed by the microcontroller unit to determine the obstacle, only in the course of reverse direction. Based on the decision made from MCU processing, the buzzer is triggered on with a specific tone of buzzing that corresponds to the obstacle's relative proximity distance with that of the wheelchair. The components that are used for implementing this Intelligent Reverse Sensor for Electric Wheelchairs are described as below:

Micro-Controller Unit - PIC16F877A [4] microcontroller incorporates several inbuilt feature like 8 channels of 10 bit Analog-to-Digital (A/D) converter, two capture/compare/PWM functions, the synchronous serial port that cane either be configured as 3-wire Serial Peripheral Interface (SPI) or the 2-wire Inter-Integrated Circuit $\left(\mathrm{I}^{2} \mathrm{C}\right)$ bus and a Universal Asynchronous Receiver Transmitter (USART). All this features have made PIC as a ideal MCU in automotive, industrial and consumer applications.

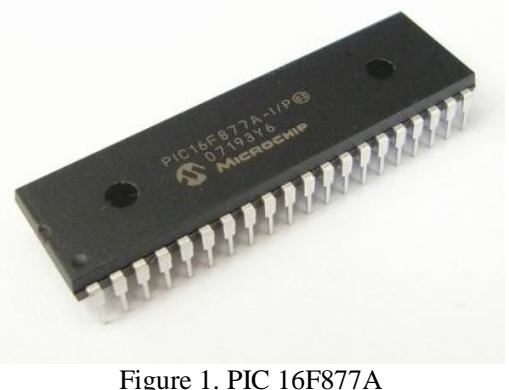

Figure 1. PIC 16F877A 
Ultrasonic range finder - Ultrasonic ranging module HCSR04 [5] provides around $2 \mathrm{~cm}$ to $400 \mathrm{~cm}$ non-contact measurement function, the ranging accuracy can reach upto $3 \mathrm{~mm}$. The module includes ultrasonic transmitter, receiver and control logic.

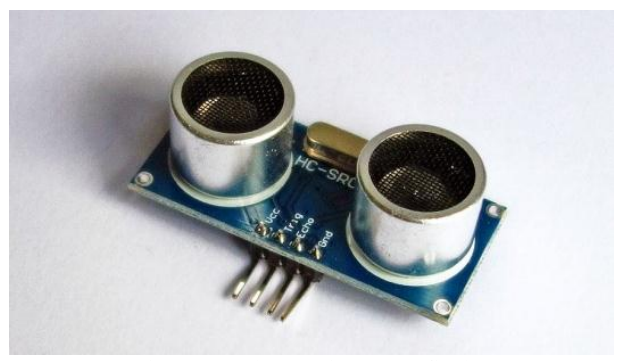

Figure 2.Ultrasonic range finder

Working - The overall system architecture is shown in Figure 3. The Intelligent Reverse Sensor for Electric Wheelchairs has a PIC16F877A microcontroller, an ultrasonic range finder and a buzzer, in addition to their existing architecture. These components are placed in a module and that is kept at rear of the wheelchair as shown in Figure 4. The ultrasonic range finder will be estimate the proximity distance between the wheelchair and the obstacle. The range thus obtained might be either increasing or decreasing based on the direction of acceleration, i.e., since the sensor module is placed in rear side of the wheelchair, the forward direction results in increasing range and the reverse direction results in decreasing range from the ultrasonic range finder.

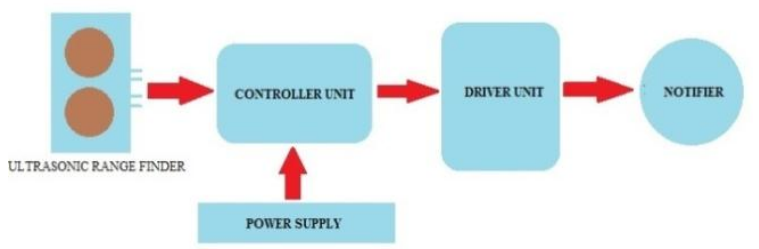

Figure 3. System architecture

The range thus obtained is fed to the PIC microcontroller and it will compare the latest pair of obtained ranges and their corresponding direction of acceleration is decided. During the forward direction (increasing range of ultrasonic sensor), no action is taken and only when the wheelchair is moving in reverse direction (decreasing range of ultrasonic sensor), that too beyond its threshold duration; it will trigger the buzzer with specific duty cycle that varies corresponding to the proximity distance to the obstacle with the aid of microcontroller.

Ensure to make that, the comparative decision making is performed since, if it is skipped, it will buzz for each and every obstacle that it encounters. Also, the threshold is in two stages and for each threshold the buzzing tone (duty cycle) is different and thus it alerts the user about the proximity level of the obstacle. Thus it results in intelligent reverse sensor for electric wheelchairs

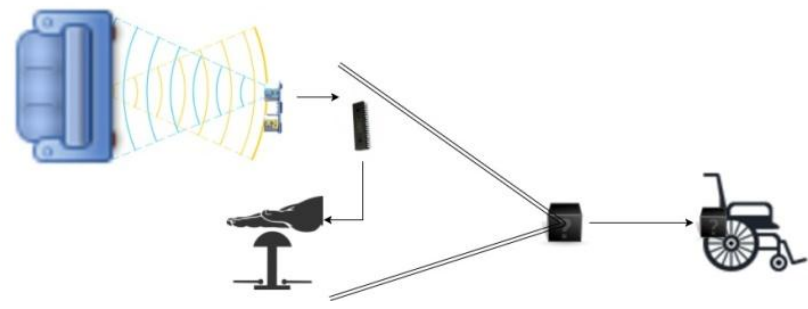

Figure 4. Functional illustration of the prototype

\section{Results AND Discussion}

Whenever the proximity distances reduces, i.e., in the reverse direction, the buzzer gets the corresponding signal from the microcontroller, as plotted in the figure, based on the level of proximity. Thus the user might be able to differentiate the distances while moving in. The prototype model is designed with two cut-off thresholds namely 20" and 35" (inches). Whenever the proximity distance crosses 35 " and still above 20 ", the buzzer will buzz alternatively; when the proximity distance is below 20", the buzzer will buzz continuously stating that the immediate attention is needed as the chance to hit the obstacle is more.

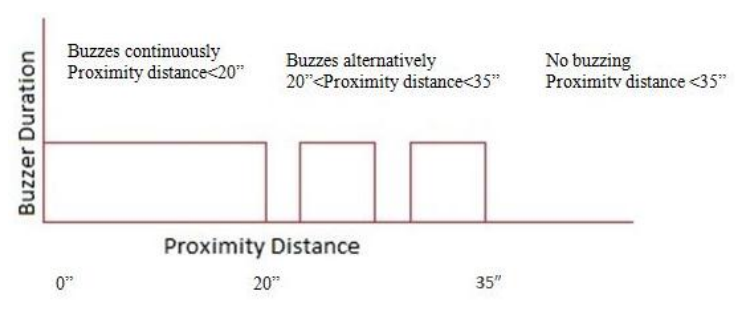

Figure 4. Buzzer Vs Proximity distance relationship

\section{CONCLUSION AND Future SCOPE}

Thus the Intelligent reverse sensor in electric wheelchair has been successfully implemented in an optimal and cost effective way. This system can be implemented in other Evehicles also thus the need for the complex algorithms can be skipped.

\section{ACKNOWLEDGEMENT}

We would like to express our heartfelt gratitude to $\mathrm{Mr}$. Venukrishnan.U, CEO of Ostrich Mobility Instruments Pvt Ltd, Bangalore, India for enthusiastic encouragement and support for this project.

We also thank all of them, who have helped and supported us to move this paper well above the level of simplicity and into 
something concrete. Finally we thank our friends, parents and relatives for giving support to do this work.

\section{REFERENCES}

[1] Disable persons in India "A statistical profile 2016"

[2] Parking sensor, Wikipedia

[3] Sunrise medical's reversing camera

[4] Microchip's PIC 16F877A datasheet

[5] Ultasonic range sensor

\section{Authors Profile}

Mr. Sooraj Surendran pursed Bachelor of Engineering in Electronics and Communication from Anna University, Chennai,India in year 2011. He is currently working as Senior Electronics Engineer, R\&D in Ostrich mobility,Bangalore,India since 2013. His main research work focuses on Electrical vehicles,

Internet of Things, etc.He has 5 years of industrial experience.

Mr. Gnanaguru $G$ pursed Master of Engineeering in Embeddded Systems from SASTRA of Thanjavur,India in year 2017. He is working as R\&D Electronics Engineer,in Ostrich mobility,Bangalore,India since 2017. His main research work focuses on Visible Light Communication, Internet of Things.

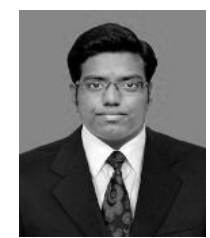

